Article

\title{
Highly efficient synthesis of benzimidazoles using microwave irradiation.
}

\author{
Monica Nardi ${ }^{1, *}$ Sonia Bonacci ${ }^{1}$, Natividad Herrera Cano ${ }^{2}$, Manuela Oliverio ${ }^{1}$ and \\ Antonio Procopio ${ }^{1}$
}

1 Dipartimento di Scienze della Salute, Università Magna Græcia, Viale Europa, Germaneto, 88100 Catanzaro CZ, Italia; monica.nardi@unicz.it (M.N.); s.bonacci@unicz.it (S.B.); nherrerac@unc.edu.ar (N.H.C.); m.oliverio@unicz,it (M. O.); procopio@unicz.it (A. P.);

2 ICYTAC, CONICET and Universidad Nacional de Córdoba, Facultad de Ciencias Químicas, Departamento. Química Orgánica. Ciudad Universitaria, Bv. Juan Filloy s/n, 5000, Córdoba, Argentina; nhc@fcq.unc.edu.ar (N.H.C.)

* Correspondence: monica.nardi@unicz.it (M.N.);

Tel. of M.N.: +39-0961-3694116

\begin{abstract}
A microwave assisted method for synthesis of 1,2-disubstituted benzimidazoles has been developed. The combination of molar ratio $N$-phenyl-o-phenylenediamine:benzaldehyde (1:1) using microwave irradiation and only $1 \%$ mol of $\operatorname{Er}(\mathrm{OTf})_{3}$ provide an efficient, environmental and mild access to a diversity of benzimidazoles under solvent-free conditions.
\end{abstract}

Keywords: microwave; green chemistry; benzimidazoles

\section{Introduction}

The use of toxic solvents in the pharmaceutical industry is is a serious problem for the environment and human health, but in the last years, Green Chemistry principles influenced the activities drug industries introducing less use of classic organic solvents [1-3], cuts in waste production with the use the recyclable reagents [4-8] and environmental organic synthetic methods.

Various research studies have been conducted on the use of "green" solvents [9-12], principally bio-solvents [13-17], ionic liquids [18-20], deep eutectic solvents [21-26], supercritical fluids [27,28], or water [29-37]. Certainly, adopting methods experimental tests based on solvent-free or solid-state reaction conditions reduces pollution. These reactions may be carried out using the reactants alone. Often the same reactions involve use of solid supports (clays, zeolites, silica, alumina or other matrices) making it easier the experimental and work-up procedures improving yield, increasing the reaction rate and lowering considerably environmental impact [38-40].

The reactions in solvent-free conditions under ultrasonic [41] or microwave [42] irradiation play a very important role in eco-sustainable extraction [43-45] and synthesis [46-53] because they greatly prevent waste and often only irradiation is useful for activating the organic reaction.

Due to their properties and applications, benzimidazoles are a class of heterocyclic compounds of great interest in the pharmaceutical chemistry area. Benzimidazole ring constitutes the basic structure of important and different pharmaceutical agents [54-58] as the vitamin $B_{12}$ [59]. For this reason, the synthesis of benzimidazoles derivatives has had considerable interest in the development of organic synthetic processes applicable on an industrial scale and with a low environmental impact. 
Recent research on the use of eco-sustainable solvents in organic chemistry for the synthesis of benzimidazoles [60-62] has had great prominence in the scientific community as well as the use of Lewis acid catalysis exploitation homogeneous catalysts [63-66] in mild reaction conditions. At the same time, experimental reactions using solid supports in conventional solvents [67-70], in green solvents [71, 72] or under solvent-free conditions [73-76] performed and the use of heterogeneous catalyst under solvent-free conditions [77] has been particularly important for the eco-sustainable synthesis of benzimidazoles.

However, the synthetic procedure for the synthesis of 1,2-disubstituted benzimidazole derivatives requires the use of MK10 20\% wt with a selectivity that is not always high. Therefore, the synthetic method has often involved the use of purification systems to obtain the desired 1,2-substituted benzimidazole derivative.

Considering our experience in Lewis acid catalysis and testing the catalytic activity of $\mathrm{Er}$ (III) in reactions under microwave irradiation [78-82] and in the synthesis of benzimidazole $[25,83]$ and benzodiazepine $[84,85]$ derivatives, we report the development of new, ecofriendly and mild method MW- assisted for the synthesis of a variety of substituted benzimidazoles. The synthetic method does not require the use of solvents but require the use of only $1 \% \operatorname{Er}(\mathrm{OTf})_{3}$ as catalyst for the formation of benzimidazole derivatives.

\section{Results}

In our initial experiment, we choose $\mathrm{N}$-phenyl-o-phenylenediamine $(1 \mathrm{mmol})$ and benzaldehyde $(1 \mathrm{mml})$ starting material in the different green solvent at different temperature to obtain selectively 1,2-disubstituted benzimidazole derivative 1a (Table 1). Initially, we tested the effect of temperature on the model reaction performing the reaction (Table 1, entry 1 ) using ethyl lactate as solvent. The reaction mixture, stirred at room temperature for $2 \mathrm{~h}$, is executed monitoring reaction by thin layer chromatography (TLC) and gas chromatography/mass spectrometry (GC/MS) analysis.

The temperature effects showed that increasing the reaction temperature to $100{ }^{\circ} \mathrm{C}$ yields are higher but insufficient (Table 1, entry 3 ).

At room temperature in waters as solvent, the GC/MS analysis showed the low conversion of the reagents within 2 hours even when increasing the temperature at $60^{\circ}$ (Table 1, entries 4 and 5). The GC-MS analysis showed the presence of the 1,2-disubstituted benzimidazole derivative with higher yields (59.6\% yields) at $60^{\circ} \mathrm{C}$ in only 120 minutes (Entry 6 in Table 1) and at a higher temperature in the same reaction time $\left(100^{\circ} \mathrm{C}, 120 \mathrm{~min}\right.$.) (Table 1, entry 7) the reaction yield increased considerably $(89.7 \%)$. When the mixture reaction subjected to microwave irradiation we obtained a good yield in only ten minutes at $60^{\circ} \mathrm{C}$ (Table 1, entry 8). Interesting was the result obtained when the reaction was carried out in (Table 1, entry 9). At this point, exploiting the activity of microwave radiation for activating the organic reactions in solvent free condition, the good conversion of $\mathrm{N}$-phenyl-o-phenilendiammine was observed obtaining the reaction product at $89.6 \%$ of yield after only 15 minutes (Table 1, entry 10). The model reaction showed the complete conversion of $\mathrm{N}$-phenyl-o-phenilendiammine when the same reaction was performed in solvent free condition at $60^{\circ} \mathrm{C}$ in only 5 minutes adding only $1 \% \operatorname{Er}(\mathrm{OTf})_{3}$ at mixture reaction (Table 1, entry 10). 
Table 1. Optimization of the reaction conditions. ${ }^{a}$

\begin{tabular}{|c|c|c|c|c|}
\hline c & Solvent & $\begin{array}{c}\text { Temp } \\
\left({ }^{\circ} \mathrm{C}\right)\end{array}$ & $\begin{array}{l}\text { Time } \\
\text { (min) }\end{array}$ & $\begin{array}{c}\text { Yield } \\
(\%)^{b}\end{array}$ \\
\hline 1 & Ethyl lactate & $\mathrm{rt}$ & 120 & 0 \\
\hline 2 & Ethyl lactate & 60 & 120 & 3.9 \\
\hline 3 & Ethyl lactate & 100 & 120 & 15.3 \\
\hline 4 & water & $\mathrm{rt}$ & 120 & 10.2 \\
\hline 5 & water & 60 & 60 & 20.9 \\
\hline 6 & water & 60 & 120 & 59.6 \\
\hline 7 & water & 100 & 120 & 89.6 \\
\hline $8^{c}$ & water & 60 & 10 & 71.9 \\
\hline 9 & - & 60 & 60 & 61.4 \\
\hline $10^{c}$ & - & 60 & 5 & 89.6 \\
\hline $11^{\mathrm{c}, \mathrm{d}}$ & - & 60 & 5 & 99.9 \\
\hline
\end{tabular}

a General reaction conditions: $N$-phenyl-o-phenylenediamine $(1 \mathrm{mmol})$ and benzaldehyde $(1$ $\mathrm{mmol}$ ) were stirred for 5-120 min at different temperatures in appropriate solvent. bPercent yield calculated from GC/MS data of the corresponding disubstituted

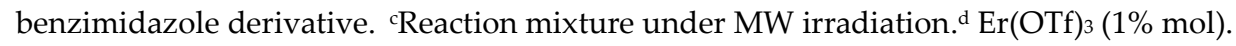

The only reagents used to obtain respective product crude in faster reaction times are been the aldehyde and the $\mathrm{N}$-phenyl-o-phenylenediamine. MW-activation for the benzimidazole formation reduces the reaction times (from $60 \mathrm{~min}$ to $5 \mathrm{~min}$ ) and enhances the yield as well (from $61.4 \%$ to $99.9 \%$ ).

At this point, the experimental procedure was applied to different aldehydes to obtain the related disubstituted benzimidazoles, and quantitative yields superior to $96 \%$ were obtained in all cases (Table 2 ).

The high-yield reaction was reported using different substituted benzaldehydes such as $p$-methyl, $p$-methoxy and $o$-hydroxy benzaldehyde (entries 2, 3 and 4, Table 2). The reactions performed with $p$-chloro, $p$-fluoro $p$-nitro benzaldehyde, aldehydes containing electron withdrawing groups, (entries 5, 6 and 7, Table 2) afford the corresponding disubstituted benzimidazoles (4a-7a) in good yields (detected by GC/MS) but in the longer reaction times (after 15 minutes).

As shown in Table 2, this new method works maintained high catalytic activity on various substituted benzaldehydes, alkyl aldehydes and cinnamaldehydes (entries 8,9 and 10). The performed reactions with $N$-benzyl o-phenylenediamines as $\mathrm{N}$-alkyl-o-phenylenediamines maintained the same reaction yield in the same condition e in the same reaction times (Table 2, entries 12, 13 and 14) 
Table 2. Synthesis of 1,2-disubstituted benzimidazoles ${ }^{a}$

\begin{tabular}{|c|c|c|c|c|}
\hline Entry & Aldehyde & Product & Time (min) & Yield $(\%)^{b}$ \\
\hline 1 & & & 5 & 99.9 \\
\hline 2 & & & 5 & 98.6 \\
\hline 3 & & & 7 & 99.6 \\
\hline 4 & & & 10 & 96.3 \\
\hline 5 & & & 15 & 96 \\
\hline 6 & & & 15 & 97 \\
\hline 7 & & & 10 & 97 \\
\hline 8 & & & 15 & 91.1 \\
\hline 9 & & & 5 & 98.2 \\
\hline
\end{tabular}


10<smiles>O=CC1CCCCC1</smiles>

11<smiles>O=C/C=C/c1ccccc1</smiles>

$12^{\mathrm{c}}$<smiles>O=Cc1ccccc1</smiles>

$13^{c}$<smiles>Cc1ccc(C=O)cc1</smiles>

$14^{c}$<smiles>COc1ccc(C=O)cc1</smiles><smiles>c1ccc(-n2c(C3CCCCC3)nc3ccccc32)cc1</smiles>

10a<smiles>c1ccc(CCc2nc3ccccc3n2-c2ccccc2)cc1</smiles>

$11 a$<smiles>c1ccc(Cn2c(-c3ccccc3)nc3ccccc32)cc1</smiles>

$1 b$

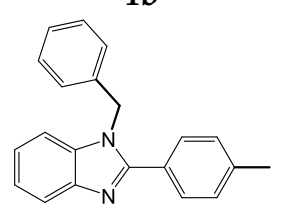

$2 b$

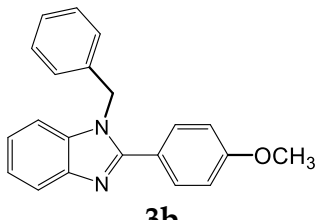

${ }^{a}$ General reaction conditions: The mixture reaction $(1 \mathrm{mmol}$ of $\mathrm{N}$-phenyl-o-phenylenediamine, $1 \mathrm{mmol}$ of aldehyde and $1 \% \mathrm{mmol}$ of $\mathrm{Er}(\mathrm{OTf})_{3}$ ) conducted in a Syntos 3000 microwave oven (Anton-Paar) at $60^{\circ} \mathrm{C}$ for 5-10 $\mathrm{min}$. The reaction mixture giving the corresponding products 1a-8a. b Percent yield calculated from GC/MS data. 'The mixture reaction conducted in the same reaction conditions using the $N-$ benzyl-o-phenylenediamine as $\mathrm{N}$-sostituited-o-phenylenediamine.

The use of the irradiation microwave has made the reaction process even more green than the previously methodologies, for faster reaction times and for greater selectivity of product formation. In the development of a green synthetic procedure, the isolation of the product is additional significant point. In our method, the benzimidazole derivatives could easily be isolated by simple addition of water and extraction with ethyl acetate, a green solvent.

Then, we also found it necessary to demonstrate the potential industrial applicability of this eco-friendly procedure, the preliminary reaction to give 1a was carried out in a large scale (20 mmol of $\mathrm{N}$-phenyl-o-phenylenediamine and $20 \mathrm{mmol}$ of benzaldehyde). The reaction was completed in 25 min with excellent yield (93\%) after simple water addition and extraction with ethyl acetate.

In conclusion, we reported a green and simple procedure for the synthesis of benzimidazoles. All reactions performed in short reaction times (5-10minutes) and with reaction yields of 86 to $99 \%$ (Table 2).

Unlike the methods reaction reported in the literature, the procedure described does not require for the use of solvents but only microwave irradiation to perform the 
complete reaction process. The proposed method reduces reaction time and energy consumption, developing the process industrially appropriate

\section{Materials and Methods}

\subsection{General Methods}

All reactions were monitored by GC-MS Shimadzu workstation. It is constituted by a GC 2010 (equipped with a 30 m-QUADREX 007-5MS capillary column, operating in the "split" mode, $1 \mathrm{~mL}$ min-1 flow of He as carrier gas).

${ }^{1} \mathrm{H}-\mathrm{NMR}$ and ${ }^{13} \mathrm{C}-\mathrm{NMR}$ spectra were recorded at $300 \mathrm{MHz}$ and at $75 \mathrm{MHz}$ respectively, using a Bruker WM 300 system. The samples solubilized in $\mathrm{CDCl}_{3}$ using tetramethylsilane (TMS) as reference $(\delta 0.00)$. Chemical shifts are given in parts per million (ppm) and coupling constants (J) are given in hertz. For ${ }^{13} \mathrm{C}-\mathrm{NMR}$ the chemical shifts are relative to $\mathrm{CDCl}_{3}(\delta 77.0)$.

Synthos 3000 instrument from Anton Paar, equipped with a $4 \times 24$ MG5 Rotor, used for the MW-assisted reactions. An external IR sensor monitors the temperature at the base of each reaction vessel.

3.2 General Procedure for the Synthesis of 1-phenyl-2-Aryl(alkyl) Benzimidazoles 1a-11a.

The aryl o alkyl aldehyde $(1 \mathrm{mmol})$ was added to the $\mathrm{N}$-phenil-o-phenilendiammine $(1 \mathrm{mmol})$ and $\operatorname{Er}(\mathrm{OTf})_{3}(1 \% \mathrm{~mol})$. The obtained mixture reaction was reacted for $5 \mathrm{~min}$ under microwave assisted, at a temperature of $60^{\circ} \mathrm{C}$ (IR Limit). After completion conversion of $\mathrm{N}$-phenil-o-phenilendiammine, the $\operatorname{Er}(\mathrm{OTf})_{3}$ was separated from the reaction mixture adding water and extracting the organic product with ethyl acetate $(4 \times 3 \mathrm{~mL})$. The products were isolated after organic phases dried over $\mathrm{Na}_{2} \mathrm{SO}_{4}$, followed by evaporation under reduced pressure. Spectral data were in accordance with the literature [86]

\subsection{General Procedure for the Synthesis of 1- benzyl-2-Aryl-Benzimidazoles 1b-3-b.}

The benzaldehyde or $p$-sostituited-benzaldehyde $(1 \mathrm{mmol})$ was added to the $\mathrm{N}$ benzyl-o-phenilendiammine $(1 \mathrm{mmol})$ and $\operatorname{Er}(\mathrm{OTf})_{3}(1 \% \mathrm{mmol})$. The obtained mixture reaction was reacted in the same reaction conditions previously reported (MW irradiation for $5 \mathrm{~min}$ ). After completion conversion of $\mathrm{N}$-phenil-o-phenilendiammine, the $\operatorname{Er}(\mathrm{OTf})_{3}$ was separated from the reaction mixture adding water and extracting the organic product with ethyl acetate $(4 \times 3 \mathrm{~mL})$. The products were isolated after organic phases dried over $\mathrm{Na}_{2} \mathrm{SO}_{4}$, followed by evaporation under reduced pressure. Spectral data were in accordance with the literature [87].

\section{Conclusions}

In summary, the current research proved a rapid, cheap, clean and environmentally sustainable method microwave assisted for the synthesis of 1,2-bisubstituted benzimidazoles. The procedure does not require the use of solvent and the simple product recovery.

The use of the Lewis catalyst $\operatorname{Er}(\mathrm{OTf})_{3}(1 \% \mathrm{mmol})$ provides a synthetic procedure which considerably reduces reaction times and waste reaction, further promoting the green chemistry principles and industrial application.

Supplementary Materials: The following are available online at www.mdpi.com/link.

Author Contributions: M.N. conceived and designed the experiments; S.B. performed the experiments; N. H. C. and M.O. analyzed the data; M. N. and A.P. wrote the paper.

Conflicts of Interest: The authors declare no conflict of interest. 


\section{References}

1. W. M. Nelso, Green Solvents for Chemistry Perspectives and Practice, Oxford University Press, 2004

2. K. Mikami, Green Reaction Media in Organic Synthesis, Blackwell, 2005.

3. Clark, J. H.; Tavener, S. J. Alternative Solvents: Shades of Green Org. Process Res. Dev. 2007, $11,1,149-155$

4. Ballini, R.; Bosica, G.; Carloni, L.; Maggi, R.; Sartori, G. Zeolite HSZ-360 as a new reusable catalyst for the direct acetylation of alcohols and phenols under solventless conditions. Tetrahedron Lett. 1998, 39, 6049-6052

5. Procopio, A.; De Luca, G.; Nardi, M.; Oliverio, M.; Paonessa, R. General MW-assisted grafting of MCM-41: Study of the dependence on time dielectric heating and solvent. Green Chem., 2009, 11, 770-773.

6. Procopio, A.; Cravotto, G.; Oliverio, M.; Costanzo, P.; Nardi, M.; Paonessa,. R. An Eco-Sustainable Erbium(III)-Catalysed Method for Formation/Cleavage of O-tert-butoxy carbonates. Green Chem., 2011, 13, 436-443.

7. Oliverio, M.; Costanzo, P.; Macario, A.; De Luca, G.; Nardi, M.; Procopio. A. A Bifuctional Heterogeneous Catalyst Erbium-Based: A Cooperative Route Towards C-C Bond Formation. Molecules, 2014, 19, 10218-10229.

8. Procopio A., Das G., Nardi M., Oliverio, M., Pasqua, L.; A Mesoporous Er(III)-MCM-41 Catalyst for the Cyanosilylation of Aldehydes and Ketones under Solvent-free Conditions. ChemSusChem, 2008, 1, 916-919.

9. W. M. Nelso, Green Solvents for Chemistry Perspectives and Practice, Oxford University Press, 2004

10. Nardi, M.; Oliverio, M.; Costanzo, P.; Sindona, G.; Procopio, A. Eco-friendly stereoselective reduction of $\alpha, \beta$-unsaturated carbonyl compounds by $\operatorname{Er}(\mathrm{OTf})_{3} / \mathrm{NaBH}_{4}$ in 2-MeTHF. Tetrahedron 2015, 71, 1132-1135.

11. Nardi, M.; Herrera Cano, N.; De Nino, A.; Di Gioia, M.L.; Maiuolo, L.; Oliverio, M.; Santiago, A.; Sorrentino, D.; Procopio, A. An eco-friendly tandem tosylation/Ferrier $\mathrm{N}$-glycosylation of amines catalyzed by $\operatorname{Er}(\mathrm{OTf})_{3}$ in 2-MeTHF. Tetrahedron Lett. 2017, 58, $1721-1726$.

12. Nardi, M.; Di Gioia, M.L.; Costanzo, P.; De Nino, A.; Maiuolo, L.; Oliverio, M.; Olivito, F.; Procopio, A. Selective acetylation of small biomolecules and their derivatives catalyzed by Er(OTf)3, Catalysts, 2017, 7, 269-282.

13. Lapkin, A.; Plucinski, P.K.; Cutler, M. Comparative assessment of technologies for extraction of artemisinin. J. Nat. Prod. 2006, 69, 1653-1664.

14. Pereira, C. S. M.; Silva, V. M. T. M.; Rodrigues, A. E. Ethyl lactate as a solvent: Properties, applications and production processes. Green Chem., 2011, 13, 2658-2671.

15. García, J. I.; García-Marín, H.; Pires, E. Glycerol based solvents: synthesis, properties and applications. Green Chem., 2014, 16, 1007-1033

16. Nardi, M.; Oliverio, M.; Costanzo, P.; Sindona, G.; Procopio, A. Eco-friendly stereoselective reduction of $\alpha, \beta$-unsaturated carbonyl compounds by $\operatorname{Er}(\mathrm{OTf})_{3} / \mathrm{NaBH}_{4}$ in 2-MeTHF. Tetrahedron 2015, 71, 1132-1135.

17. Nardi, M.; Herrera Cano, N.; De Nino, A.; Di Gioia, M.L.; Maiuolo, L.; Oliverio, M.; Santiago, A.; Sorrentino, D.; Procopio, A. An eco-friendly tandem tosylation/Ferrier $\mathrm{N}$-glycosylation of amines catalyzed by $\operatorname{Er}(\mathrm{OTf})_{3}$ in 2-MeTHF. Tetrahedron Lett. 2017, 58, 1721-1726.

18. Abbott, A.P.; Davies, D.L.; Capper, G.; Rasheed, R.K.; Tambyrajah, V. Ionic Liquids and Their Use As solvents. U.S. Patent, 7,183,433, 27 February 2007.

19. Di Gioia, M.L.; Costanzo, P.; De Nino, A.; Maiuolo, L.; Nardi, M.; Olivito, F., Procopio, A. Simple and efficient Fmoc removal in ionic liquid. RSC Adv. 2017, 7, 36482-36491

20. De Nino, A.; Maiuolo, L.; Merino, P.; Nardi, M.; Procopio, A.; Roca-Lõpez, D.; Russo, B.; Algieri, V. Efficient organocatalyst supported on a simple ionic liquid as a recoverable system for the asymmetric diels-alder reaction in the presence of water, ChemCatChem, 2015, 7, 5, 830-835

21. Abbott, A.P.; Capper, G.; Davies, D.L.; Rasheed, R.K.; Tambyrajah, V. Novel solvent properties of choline chloride/urea mixtures. Chem. Commun. 2003, 70-71.

22. Gorke, J.T.; Srienc, F.; Kazlauskas, R.J. Hydrolase-catalyzed biotransformations in deep eutectic solvents. Chem. Commun. 2008, 10, 1235-1237. 
23. Smith, E.L.; Abbott, A.P.; Ryder, K.S. Deep Eutectic Solvents (DESs) and their applications Chem. Rev. 2014, 114, 11060-11082.

24. Paiva, A.; Craveiro, R.; Aroso, I.; Martins, M.; Reis, R.L.; Duarte A.R.C. Natural deep eutectic solvents-Solvents for the 21st century. ACS Sustainable Chem. Eng. 2014, 2, 1063-1071.

25. Di Gioia, M.L.; Cassano, R.; Costanzo, P.; Herrera Cano, N.; Maiuolo, L.; Nardi, M.; Nicoletta, F. P.; Oliverio, M.; Procopio, A. Green Synthesis of Privileged Benzimidazole Scaffolds Using Active Deep Eutectic Solvent Molecules 2019, 24(16), 2885

26. Bonacci, S.; Di Gioia, M.L.; Costanzo, P.; Maiuolo, L.; Tallarico, S.; Nardi, M. Natural Deep Eutectic Solvent as Extraction Media for the Main Phenolic Compounds from Olive Oil Processing Wastes Antioxidants 2020, 9(6), 513;

27. Leitner, W.; Poliakoff, M. Supercritical fluids in green chemistry Green Chem., 2008, 10, 730-730

28. Carlès P. A brief review of the thermophysical properties of supercritical fluids $J$. of Supercritical Fluids 2010, 53, 2-11

29. Lindström, U.M. Stereoselective Organic Reactions in Water. Chem. Rev. 2002, 10, 2751-2772.

30. Procopio, A.; Gaspari, M.; Nardi, M.; Oliverio, M.; Tagarelli, A.; Sindona, G. Simple and efficient MW-assisted cleavage of acetals and ketals in pure water. Tetrahedron Lett. 2007, 48, 8623-8627.

31. Procopio, A.; Gaspari, M.; Nardi, M.; Oliverio, M.; Rosati, O. Highly efficient and versatile chemoselective addition of amines to epoxides in water catalyzed by erbium(III) triflate Tetrahedron Lett , 2008, 49, 2289-2293.

32. Simon, M.O.; Li, C.J. Green chemistry oriented organic synthesis in water. Chem. Soc. Rev. 2012, 41, 1415-1427.

33. Oliverio, M., Costanzo, P., Paonessa, R., Nardi, M., Procopio, A. Catalyst-free tosylation of lipophilic alcohols in water RSC Advances, 2013, 3(8), 2548-2552

34. Nardi, M.; Herrera Cano, N.; Costanzo, P.; Oliverio, M.; Sindona, G.; Procopio, A. Aqueous MW eco-friendly protocol for amino group protection. RSC Adv. 2015, 5, 18751-18760.

35. Nardi, M.; Di Gioia, M.L.; Costanzo, P.; De Nino, A.; Maiuolo, L.; Oliverio, M.; Olivito, F.; Procopio, A. Selective acetylation of small biomolecules and their derivatives catalyzed by Er(OTf) 3, Catalysts, 2017, 7, 269-282.

36. Nardi, M.; Costanzo, P.; De Nino, A.; Di Gioia, M.L.; Olivito, F.; Sindona, G.; Procopio, A. Water excellent solvent for the synthesis of bifunctionalized cyclopentenones from furfural, Green Chem., 2017, 19, 5403-5411.

37. Olivito, F.; Costanzo, P.; Di Gioia, M.L.; Nardi, M.; Oliverio, M.; Procopio, A. Efficient synthesis of organic thioacetate in water. Org. Biomol. Chem., 2018, 16, 7753-7759.

38. Procopio, A.; De Luca, G.; Nardi, M.; Oliverio, M.; Paonessa, R. General MW-assisted grafting of MCM-41: Study of the dependence on time dielectric heating and solvent Green Chem., 2009, 11, 770-773.

39. Estevão, M.S.; Afonso, C.A.M. Synthesis of trans-4,5-diaminocyclopent-2-enones from furfural catalyzed by Er(III) immobilized on silica, Tetrahedron Letters 2017, 58, 302-304

40. Senthilkumar, S.; Maru M. S.; Somani, R. S.; Bajaj, H. C.; Neogi. S. Unprecedented $\mathrm{NH}_{2}-\mathrm{MIL}-101(\mathrm{Al}) / n$-Bu4NBr system as solvent-free heterogeneous catalyst for efficient synthesis of cyclic carbonates via $\mathrm{CO}_{2}$ cycloaddition Dalton Trans., 2018,47, 418-428.

41. T. J. Mason Sonochemistry: current uses and future prospects in the chemical and processing industries Phil. Trans. R. Soc. Lond. A, 1999, 357, 355-369

42. A. Loupy Solvent-free microwave organic synthesis as an efficient procedure for green chemistry C. R. Chimie 2004, 7, 103-112

43. Nardi, M.; Bonacci, S.; De Luca, G.; Maiuolo, J.; Oliverio, M.; Sindona, G.; Procopio, A. Biomimetic synthesis and antioxidant evaluation of 3,4-DHPEA-EDA [2-(3,4-hydroxyphenyl) ethyl (3S,4E)-4-formyl-3-(2-oxoethyl)hex-4-enoate], Food Chemistry, 2014, 162, 89-93.

44. Nardi, M.; Bonacci, S.; Cariati, L.; Costanzo, P.; Oliverio, M.; Sindona, G.; Procopio, A. Synthesis and antioxidant evaluation of lipophilic oleuropein aglycone derivatives Food and Function, 2017, 8, 12, 4684-4692

45. Costanzo, P.; Bonacci, S.; Cariati, L.; Nardi, M.; Oliverio, M.; Procopio, A. Simple and efficient sustainable semi-synthesis of oleacein [2-(3,4-hydroxyphenyl) ethyl 
(3S,4E)-4-formyl-3-(2-oxoethyl)hex-4-enoate] as potential additive for edible oils Food Chemistry, 2018, 245, 410-414.

46. Oliverio, M.; Costanzo, P.; Nardi, M.; Calandruccio, C.; Salerno, R.; Procopio, A. Tunable microwave-assisted method for the solvent-free and catalyst-free peracetylation of natural products. Beilstein. J. Org. Chem. 2016, 12, 2222-2233.

47. Maiuolo, L.; Merino, P.; Algieri, V.; Nardi, M.; Di Gioia, M.L.; Russo, B.' Delso, I.; Tallarida, M.A.; De Nino, A. Nitrones and nucleobase-containing spiro-isoxazolidines derived from isatin and indanone: Solvent-free microwave-assisted stereoselective synthesis and theoretical calculations RSC Adv. 2017, 7, 48980-48988.

48. Bortolini, O.; D'Agostino, M.; De Nino, A.; Maiuolo, L.; Nardi, M.; Sindona, G. Solvent-free, microwave assisted 1,3-cycloaddition of nitrones with vinyl nucleobases for the synthesis of N,O-nucleosides Tetrahedron, 2008, 64(35), 8078-8081

49. Procopio, A.; Gaspari, M.; Nardi, M.; Oliverio, M.; Romeo, R. MW-assisted Er(OTf) 3 -catalyzed mild cleavage of isopropylidene acetals in Tricky substrates Tetrahedron Letters, 2008, 49(12), 1961-1964.

50. Maiuolo, L.; De Nino, A.; Algieri, V.; Nardi, M. Microwave-assisted 1,3-dipolar cyclo-addition: Recent advances in synthesis of isoxazolidines Mini-Reviews in Organic Chemistry 2017, 14(2), 136-142.

51. Paonessa, R.; Nardi, M.; Di Gioia, M.L.; Olivito, F.; Oliverio, M., Procopio, A. Eco-friendly synthesis of lipophilic EGCG derivatives and antitumor and antioxidant evaluation Natural product communications, 2018, 9, 1117-1122

52. Costanzo, P.; Calandruccio, C.; Di Gioia, M.L.; Nardi, M.; Oliverio, M.; Procopio, A. First multicomponent reaction exploiting glycerol carbonate synthesis, Journal of Cleaner Production, 2018, 202, 504-509

53. Oliverio, M.; Nardi, M.; Cariati, L.; Vitale, E.; Bonacci, S.; Procopio, A. "on Water" MW-Assisted Synthesis of Hydroxytyrosol Fatty Esters ACS Sustainable Chem. Eng. 2016, 4, 661-665

54. Kubo, K.; Oda, K.; Kaneko, T.; Satoh, H.; Nohara, A. Synthesis of 2-(4Fluoroalkoxy-2-pyridyl) methyl] sulfinyl]-1H-benzimidazoles as Antiulcer Agents. Chem Pharm Bull. 1990, 38(10), 2853-2858.

55. Uchida, M.; Chihiro, M.; Morita, S.; Yamashita, H.; Yamasaki, K.; Kanbe, T.; Yabuuchi, Y.; Nakagawz, K. Synthesis and Antiulcer Activity of 4- Substituted 8-[(2-Benzimidazolyl) sulfinylmethyl]-1, 2, 3, 4-tetrahydroquinolines and Related Compounds. Chem Pharm Bull. 1990, 38(6), 1575-1586.

56. Grassi, A.; Ippen, J.; Bruno, M.; Thomas, G.; Bay, P. A thiazolylamino benzimidazole derivative with gastroprotective properties in the rat. Eur J Pharmacol. 1991, 195(2), 251-259.

57. Ozkay, Y.; Tunali, Y.; Karaca, H.; Isikdag I. Antimicrobial activity and a SAR study of some novel benzimidazole derivatives bearing hydrazones moiety. Eur J Med Chem 2010, 45(8), 3293-3298.

58. Algul, O.; Karabulut, A.; Canacankatan, N.; Gorur, A.; Sucu, N.; Vezir, O Apoptotic and anti-angiogenic effects of benzimidazole compounds: relationship with oxidative stress mediated ischemia/reperfusion injury in rat hind limb Antiinflamm Antiallergy Agents Med Chem. 2012; 11(3, 267-75.

59. Emerson, G.; Brink, N. G.; Holly, F. W.; Koniuszy, F.; Heyl, D.; Folker, K. Vitamin B12 . VIII. Vitamin $B_{12}$-Like Activity of 5,6-Dimethylbenzimidazole and Tests on related compounds. J. Am. Chem. Soc., 1950, 72, 3084-3085

60. Kommi, D.N.; Jadhavar, P.S.; Kumar, D.; Chakraborti, A.K. "All-water" one-pot diverse synthesis of 1,2-disubstituted benzimi dazoles: Hydrogen bond driven synergistic electrophile-nucleophile dual activation by water. Green Chem. 2013, 15, 798-810.

61. Gioia, M.L.D.; Cassano, R.; Costanzo, P.; Cano, N.H.; Maiuolo, L.; Nardi, M.; Nicoletta, F.P.; Oliverio, M.; Procopio, A. Green synthesis of privileged benzimidazole scaffolds using active deep eutectic solvent. Molecules 2019, 24, 2885.

62. Shen, M.-G.; Cai, C. Ytterbium perfluorooctanesulfonates catalyzed synthesis of benzimidazole derivatives in fluorous solvents. J. Fluor. Chem. 2007, 128, 232-235.

63. Jayabharathi, J.; Thanikachalam, V.; Jayamoorthy, K. Synthesis of some fluorescent benzimidazole derivatives using cobalt(II) hydroxide as highly efficient catalyst-spectral and physico-chemical studies and ESIPT process. Photochem. Photobiol. Sci. 2013, 12, 1761-1773. 
64. Srinivasulu, R.; Kumar, K.R.; Satyanarayana, P.V.V. Facile and Efficient Method for Synthesis of Benzimidazole Derivatives Catalyzed by Zinc Triflate Green Sustainable Chem. 2014, 4, 33-37.

65. Martins, G.M.; Puccinelli, T.; Gariani, R.A.; Xavier, F.R.; Silveira, C.C.; Mendes, S.R. Facile and efficient aerobic one-pot synthesis of benzimidazoles using $\mathrm{Ce}\left(\mathrm{NO}_{3}\right)_{3} \cdot 6 \mathrm{H}_{2} \mathrm{O}$ as promoter. Tetrahedron Lett. 2017, 58, 1969-1972.

66. Peng, X.-C.; Gong, S.-S.; Zeng, D.-Y.; Duo, S.-W.; Sun, Q. Activated carbon supported hafnium(IV) chloride as an efficient, recyclable, and facile removable catalyst for expeditious parallel synthesis of benzimidazoles. Catalysts 2020, 10, 436.

67. Procopio, A.; De Luca, G.; Nardi, M.; Oliverio, M.; Paonessa, R. General MW-assisted grafting of MCM-41: Study of the dependence on time dielectric heating and solvent. Green Chem., 2009, 11, 770-773.

68. Sontakke, VA.; Ghosh, S.; Lawande, P.P.; Chopade, B.A.; Shinde, V.S. A simple, efficient synthesis of 2-aryl benzimidazoles using silica supported periodic acid catalyst and evaluation of anticancer activity. ISRN Organic Chemistry. 2013, 1-7.

69. Kumar, K.R.; Satyanarayana, P.V.V.; Reddy, BS. $\mathrm{NaHSO}_{4}-\mathrm{SiO}_{2}$ promoted synthesis of benzimidazole derivatives. Archives of Applied Science Research. 2012, 4, 1517-1521

70. Goswami, M.; Dutta, M.M.; Phukan, P. Sulfonic-acid-functionalized activated carbon made from tea leaves as green catalyst for synthesis of 2-substituted benzimidazole and benzothiazole. Res Chem Intermed, 2018, 44, 1597-1615

71. Dhakshinamoorthy, A.; Kanagaraj, K.; Pitchumani, K. Zn2+-K10-clay (clayzic) as an efficient water-tolerant, solid acid catalyst for the synthesis of benzimidazoles and quinoxalines at room temperature. Tetrahedron Lett. 2011, 52, 69-73.

72. Bonacci, S-; Nardi, M.; Costanzo, P.; De Nino, A.; Di Gioia, M.L.; Oliverio, M.; Procopio, A. Montmorillonite K10-Catalyzed Solvent-Free Conversion of Furfural into Cyclopentenones Catalysts, 2019, 9, 301-311.

73. Procopio A., Das G., Nardi M., Oliverio, M., Pasqua, L.; A Mesoporous Er(III)-MCM-41 Catalyst for the Cyanosilylation of Aldehydes and Ketones under Solvent-free Conditions. ChemSusChem, 2008, 1, 916-919.

74. Procopio, A.; De Nino, A.; Nardi, M.; Oliverio, M.; Paonessa, R.; Pasceri, R. A New Microwave-Assisted Organocatalytic Solvent-Free Synthesis of Optically Enriched Michael Adducts Synlett 2010, 12, 1849-1853.

75. Procopio, A.; Cravotto, G.; Oliverio, M.; Costanzo, P.; Nardi, M.; Paonessa,. R. An Eco-Sustainable Erbium (III)-Catalysed Method for Formation/Cleavage of O-tert-butoxy carbonates. Green Chem., 2011, 13, 436-443.

76. Oliverio, M.; Costanzo, P.; Macario, A.; De Luca, G.; Nardi, M.; Procopio. A. A Bifuctional Heterogeneous Catalyst Erbium-Based: A Cooperative Route Towards C-C Bond Formation. Molecules, 2014, 19, 10218-10229.

77. Bonacci, S.; Iriti, G.; Mancuso, S.; Novelli, P.; Paonessa, R.; Tallarico, S.; Nardi, M. Montmorillonite K10: An efficient organheterogeneous catalyst for synthesis of benzimidazole derivatives. Catalysts 2020, 10, 84

78. Procopio A.; Dalpozzo R.; De Nino A.; Maiuolo L.; Nardi M.; Romeo G. Mild and efficient method for the cleavage of benzylidene acetals by using erbium (III) triflate Org. Biomol. Chem. 2005, 3, 22, $4129-4133$

79. Procopio, A.; Celia, C.; Nardi, M.; Oliverio, M.; Paolino, D.; Sindona, G. Lipophilic hydroxytyrosol esters: Fatty acid conjugates for potential topical administration Procopio A. J. Nat. Prod. 2011, 74, 11, 2377 - 2381

80. Nardi, M.; Bonacci, S.; De Luca, G.; Maiuolo, J.; Oliverio, M.; Sindona, G.; Procopio, A. Biomimetic synthesis and antioxidant evaluation of 3,4-DHPEA-EDA [2-(3,4-hydroxyphenyl) ethyl (3S,4E)-4-formyl-3-(2-oxoethyl)hex-4-enoate], Food Chemistry, 2014, 162, 89-93.

81. Nardi, M.; Bonacci, S.; Cariati, L.; Costanzo, P.; Oliverio, M.; Sindona, G.; Procopio, A. Synthesis and antioxidant evaluation of lipophilic oleuropein aglycone derivatives Food and Function, 2017, 8, 12, 4684-4692

82. Paonessa, R.; Nardi, M.; Di Gioia, M.L.; Olivito, F.; Oliverio, M., Procopio, A. Eco-friendly synthesis of lipophilic EGCG derivatives and antitumor and antioxidant evaluation Nat. Prod. Commun., 2018, 9, 1117-1122 
83. Herrera Cano, N.; Uranga, J.G.; Nardi, M.; Procopio, A.; Wunderlin, D.A.; Santiago, A.N. Selective and eco-friendly procedures for the synthesis of benzimidazole derivatives. The role of the $\mathrm{Er}(\mathrm{OTf}) 3$ catalyst in the reaction selectivity, Beilstein J. Org. Chem. 2016, 12, 2410-2419.

84. Nardi, M.; Cozza, A.; Maiuolo, L.; Oliverio, M.; Procopio, A. 1,5-Benzoheteroazepines through eco-friendly general condensation reactions Tetrahedron Letters, 2011, 52 (38), 4827-4834.

85. Nardi, M.; Cozza, A.; De Nino, A.; Oliverio, M.; Procopio, A. One-pot synthesis of dibenzo[b,e][1,4]diazepin-1-ones. Synthesis 2012, 44, 800-804.

86. Zhong, R.; Xiong, W.; Zhang, H.; Zeng, T.; Gong, S.; Sun, Q. Highly Efficient and Ambient Temperature Synthesis of Benzimidazoles via Co(III)/Co(II)- Mediated Redox Catalysis. Catalysts 2022, 12, 59.

87. Mamedov, V. A.; Zhukova, N. A. Recent Developments Towards Synthesis of (Het)arylbenzimidazoles Synthesis 2021, 53, 1849-1878. 